\title{
Infecciones de transmisión sexual en mujeres de edad fértil usuarias del Hospital Regional de Villa Hayes, Paraguay
}

\author{
Sandra Virginia Caballero Gómez ${ }^{\text {, }}$ Adriana Beatriz Valenzuela Cáceres ${ }^{\text {II }}$, Santiago Evers Orihuela ${ }^{\text {III }}$, \\ Laura Patricia Mendoza Torres ${ }^{\text {II }}$ \\ I. Facultad de Ciencias Químicas, Universidad Nacional de Asunción, San Lorenzo, Paraguay \\ II. Instituto de Investigaciones en Ciencias de la Salud, Universidad Nacional de Asunción, San Lorenzo, Para- \\ guay \\ III. Hospital Regional de Villa Hayes, MSP-BS, Villa Hayes, Paraguay
}

Cómo referenciar este artículo/ How to reference this article:
Caballero S, Valenzuela A, Evers S, Mendoza L. Infecciones de transmisión sexual en mujeres de edad fértil usuarias del Hospital Regional de Villa Hayes, Paraguay. Mem. Inst. Investig. Cienc. Salud, Agosto 2015; Vol. 13(2):39-49

\begin{abstract}
RES U M E N
Las Infecciones de Transmisión Sexual (ITS) se encuentran entre las cinco razones de consulta más frecuente en adultos. En Paraguay existe un conocimiento limitado sobre frecuencia de ITS especialmente en mujeres no gestantes en edad fértil. Por ello, este estudio piloto descriptivo de corte transverso analizó la frecuencia de sífilis, de infección por el Virus de la Inmunodeficiencia Humana (VIH) de factores de riesgo asociados en 100 mujeres en edad fértil (18-48 años) no gestantes que acudieron al Hospital Regional de Villa Hayes. La detección de sífilis se realizó mediante el método inmunocromatográfico y la confirmación por la Prueba de Absorción de Anticuerpos Treponémicos Fluorescentes. La infección por VIH fue detectada mediante el método inmunocromatográfico y el Ensayo por Inmunoabsorción Ligado a Enzimas. Se consideró un conocimiento aceptable sobre prevención y transmisión de estas ITS si el $60 \%$ de las preguntas se contestaban correctamente. Se observó una alta frecuencia de sífilis, de $13 \%$ (IC $95 \% 7,1$ $-21,2 \%)$ y una asociación entre el bajo nivel educativo y la presencia de sífilis $(p=0,002)$. No se encontró ningún resultado positivo para VIH. El $11 \%$ de las mujeres presentó un nivel inaceptable de conocimiento, sin embargo se evidenció una alta frecuencia de mujeres ( $58 \%$ ) que no utilizaba preservativo, lo cual indica falta de buenas prácticas. Es preciso fortalecer las medidas de control y manejo de ITS ya existentes en esta región especialmente en las mujeres no gestantes en edad fértil.
\end{abstract}

Palabras clave: Sífilis, VIH, factores de riesgo, conocimiento, mujeres en edad fértil.

\section{Sexually transmitted infections in childbearing age women attending at the Regional Hospital of Villa Hayes, Paraguay}

\section{A B S T R A C T}

Sexually Transmitted Infections (STIs) are among the five most frequent reasons for consultation in adults. In Paraguay there is a limited knowledge about STIs frequency especially in non pregnant women of childbearing age. Therefore, this pilot descriptive cross-sectional study analyzed the frequency of syphilis, HIV infection and associated risk factors in 100 women of childbearing age (18-48 years) non pregnant who went to the Regional Hospital of Villa Hayes. Syphilis screening was performed using the immu- 
nochromatography method and confirmation by the Test Fluorescent Treponemal Antibody Absorption. HIV infection was detected by the immunochromatographic method and the test for enzyme-linked immunosorbent. An acceptable knowledge about prevention and transmission of these STIs were considered if $60 \%$ of the questions were answered correctly. A high frequency of syphilis, about $13 \%\left(\mathrm{CI}_{95 \%} 7.1-21.2 \%\right)$ and an association between low educational level and the presence of syphilis $(p=0.002)$ was observed. No positive result for HIV was found. $11 \%$ of women presented an unacceptable level of knowledge, however a high frequency of women (58\%) who did not use condoms was evident, indicating lack of good practices. It is necessary to strengthen control measures and management of STIs existing in this region especially in nonpregnant women of childbearing age.

Key words: syphilis, HIV, risk factors, knowledge, women of childbearing age.

\section{INTRODUCCIÓN}

La prevalencia e incidencia de las Infecciones de Transmisión Sexual (ITS) entre las diversas regiones del mundo son muy diversas. Entre las ITS curables se encuentra la sífilis, en donde la mayor incidencia de esta infección se observa en África, con 3,4 millones de casos registrados en el 2008 (1); además esta región presenta uno de los niveles de prevalencia mayores de una de las ITS incurables como es la infección por el Virus de la Inmunodeficiencia Humana (VIH), con 5,6 millones de casos (2).

Según datos registrados en el 2008, eran tres los continentes que presentaban la mayor incidencia de sífilis, en donde América ocupaba el tercer lugar registrando 2,8 millones de casos (1).

Cabe resaltar que las ITS que se manifiestan con ulceras genitales, como sífilis, presentan una mayor probabilidad de riesgo a la adquisición de la infección por VIH durante relaciones sexuales sin protección en comparación con las ITS no ulcerativas (1).

En Paraguay se ha registrado en el 2013 una prevalencia de sífilis en parturientas y puérperas de $4,41 \%$ y dentro de este grupo de mujeres las más afectadas eran las que tenían edades comprendidas entre 25 a 29 años según el Programa Nacional de Control de SIDA-ITS (PRONASIDA)(3). Entre las poblaciones más vulnerables, se encuentran las Mujeres Trabajadoras Sexuales (MTS), con una prevalencia registrada en el 2012 en nuestro país de $30,37 \%$ (9).

En cuanto al VIH/SIDA, en nuestro país a finales del 2013 fueron reportados 1209 casos, en donde el $70 \%$ fueron del sexo masculino en edades comprendidas entre 25 a 30 años. No obstante en el sexo femenino la mayor incidencia se observó en el grupo de 20 a 25 años de edad (3). La prevalencia encontrada en nuestro país para las MTS en el año 2012 fue de $2,13 \%$ (4).

Actualmente la información disponible acerca de la distribución y frecuencia de las ITS en América Latina es muy limitada, y se cree que una parte de este problema es debido a la sub-notificación de los casos; por otra parte se cree que es debido a que muchas de estas infecciones cursan sin sintomatología o signo alguno favoreciendo a que la demanda de los servicios sea menor pudiendo únicamente identificarse dichos casos mediante el empleo de programas de investigación activa (5).

En un estudio reciente realizado en nuestro país en el Departamento de Presidente Hayes, se demostró que existe una alta frecuencia de sífilis y de infección por el VIH en mujeres no gestantes con inicio de relaciones sexuales en algunas comunidades indígenas (6). Por ello, el objetivo del presente estudio es determinar la frecuencia de sífilis, infección por VIH, y los factores de riesgo asociados con la adquisición de estas infecciones en mujeres no gestantes en edad fértil. 


\section{MATERIALES Y MÉTODO}

\section{Diseño y procedimiento}

Estudio piloto descriptivo de corte transverso realizado durante el mes de octubre del año 2014, llevado a cabo en 100 mujeres en edad fértil de 18 a 48 años de edad (OMS) (7) no gestantes que ya habían iniciado las relaciones sexuales y que acudieron a los consultorios clínicos, al Departamento de Planificación Familiar, al Laboratorio de Análisis Clínicos o a sus puestos de trabajo dentro del Hospital Regional de Villa Hayes (Figura 1), dependiente del Ministerio de Salud Pública y Bienestar Social (MSPyBS). No se incluyeron mujeres internadas, indígenas y aquellas que habían tenido alguna alteración de sus facultades mentales y/o que no comprendían el significado del estudio.

El proyecto fue socializado con el Director del Hospital Regional de Villa Hayes. Una vez socializado se realizó un acuerdo de colaboración científica donde la autoridad máxima del Hospital determinó los profesionales que iban a participar del proyecto. A todas las mujeres que cumplieron con los requisitos se les consultó si estaban de acuerdo de participar del estudio, además se les explicó los riesgos y beneficios del estudio. Previa a la toma de muestras firmaron un consentimiento informado y luego fueron entrevistadas respondiendo a una encuesta sobre características sociodemográficas, económicas y hábitos sexuales. Dentro de esta encuesta estaban incluidas 7 preguntas sobre prevención y transmisión de sífilis e infección por el VIH de forma a evaluar el nivel de conocimiento que poseían las mujeres, de modo que si respondían correctamente 4 de estas preguntas se consideraba que tenían un conocimiento aceptable, si respondían 5 de las preguntas tenían conocimiento bueno, 6 de las preguntas conocimiento muy bueno y las 7 preguntas conocimiento excelente (8).

Posteriormente se procedió a la extracción de muestras de $3 \mathrm{~mL}$ de sangre para la detección de los anticuerpos dirigidos contra VIH y Treponema pallidum. Todos los resultados fuera de rango fueron confirmados.

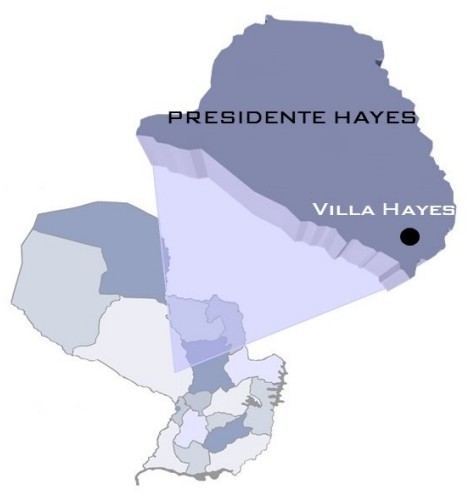

Figura 1. Ubicación del Hospital Regional de Villa Hayes en la Ciudad de Villa Hayes, Dpto. de Pdte. Hayes.

\section{Aspectos Éticos}

El protocolo de estudio fue aprobado por el Comité de Ética de la Facultad de Ciencias Químicas de la Universidad Nacional de Asunción. La identidad de cada mujer se mantuvo de manera confidencial, ya que toda la información se procesó en forma de códigos y fue almacenada en una computadora con acceso restringido a investigadores de este estudio. Los resultados fueron entregados al Hospital Regional, quienes se encargaron de informar los mismos a las mujeres participantes y monitorear los tratamientos en los casos necesarios. 


\section{Detección de sífilis}

Primeramente en el Hospital, se realizó un ensayo inmunocromatográfico de fase sólida (SD BIOLINE Syphilis 3.0, Corea) como método de screening para la detección cualitativa de anticuerpos de todos los isotipos (IgG, IgM, IgA) dirigidos contra el Treponema pallidum. Todas las muestras que dieron positivo al ensayo inmunocromatográfico fueron remitidas al Instituto de Investigaciones en Ciencias de la Salud (IICS) y los resultados confirmados por la Prueba de Absorción de Anticuerpos Treponémicos Fluorescentes (FTA-ABS, conjugado IgG, lámina Trepo-Spot IF, BioMérieux, Francia).

\section{Detección de VIH}

En el Hospital, se realizó la detección cualitativa de anticuerpos de todos los isotipos (IgG, IgM, IgA) dirigidos contra el VIH-1 y 2 simultáneamente por ensayo inmunocromatográfico (The Advanced Quality One Step Anti-HIV-1 y 2 Tri-line Test). Además en el IICS, todas las muestras fueron procesadas por el Ensayo por Inmunoabsorción Ligado a Enzimas (ELISA, Genscreen Ultra HIV Ag-Ab, Bio-Rad, Francia) a modo de detectar también antígenos VIH p24 y de anticuerpos dirigidos contra VIH-1 y VIH-2.

\section{Análisis estadístico}

El análisis de los datos se realizó empleando procedimientos de estadística descriptiva utilizando el programa Epi Info ${ }^{\mathrm{TM}} 7$ (CDC, Atlanta).

Para encontrar posibles asociaciones entre las características socio-demográficas, económicas y hábitos sexuales con la presencia de sífilis, fue utilizado el test de chi cuadrado, considerándose estadísticamente significativa los valores de $p \leq 0,05$. Las variables cuantitativas (edad, años de estudio alcanzado, ingreso económico per cápita, edad de inicio de relaciones sexuales, número de parejas sexuales) fueron convertidas a variables dicotómicas a fin de realizar el análisis por el test de chi cuadrado. El punto de corte fue seleccionado en base a los valores de las medianas observadas en la población de mujeres estudiadas. Las medianas fueron acompañadas con sus respectivos intervalos de confianza $\left(\mathrm{IC}_{25 \%-75 \%}\right.$ ) y las medias con el $\mathrm{IC}_{95 \%}$.

\section{RESULTADOS}

En cuanto a las características socio-demográficas y socio-económicas, la edad de las 100 mujeres incluidas estuvo comprendida entre 18 y 48 años, siendo la mediana de 28 años (IC $25 \%-75 \%$ 23,5-35 años). La mayoría de las mujeres estaba casada o tenía pareja $(69 \%, 69 / 100)$. El nivel de estudio secundario fue el que presentó mayor frecuencia $(42 \%, 42 / 100)$, siendo la mediana de los años de escolarización de 10 años ( IC $_{25 \%} 75 \%$, 6-13 años). El 75\% (75/100) de las mujeres vivía en áreas urbanas, y el 17\% (17/100) residía en condiciones de hacinamiento. Aproximadamente la mitad de las mujeres $(48 \%, 48 / 100)$ tenía un nivel socioeconómico bajo o muy bajo, siendo la mediana del ingreso económico per cápita de $439.286 \mathrm{Gs} / \mathrm{mes}$. Se observó que el 97\% (97/100) utilizaba al menos una vez al año los servicios del hospital. Estos datos se muestran en la Tabla 1. 
Tabla 1. Características socio-demográficas y socio-económicas de mujeres en edad fértil no gestantes. Hospital Regional de Villa Hayes. $n=100$

\begin{tabular}{|c|c|c|}
\hline $\begin{array}{l}\text { Características } \\
\end{array}$ & $\mathbf{n}$ & $\%$ \\
\hline Edad en años (mediana, $\mathrm{IC}_{25 \%-75 \%}$ ) & 28 & $23,5-35$ \\
\hline$<20$ & 10 & 10 \\
\hline $20-29$ & 47 & 47 \\
\hline $30-39$ & 29 & 29 \\
\hline$\geq 40$ & 14 & 14 \\
\hline \multicolumn{3}{|l|}{ Estado Civil } \\
\hline Casada o en pareja & 69 & 69 \\
\hline Soltera o divorciada & 31 & 31 \\
\hline Escolaridad en años (mediana, $\mathrm{IC}_{25 \%-75 \%}$ ) & 10 & $6-13$ \\
\hline Primaria & 32 & 32 \\
\hline Secundaria & 42 & 42 \\
\hline Terciaria & 26 & 26 \\
\hline \multicolumn{3}{|l|}{ Ocupación } \\
\hline Con empleo remunerado & 34 & 34 \\
\hline Sin empleo & 66 & 66 \\
\hline $\begin{array}{l}\text { Ingreso Económico Per cápita (guaraníes/mes)* } \\
\text { (mediana, } \mathrm{IC}_{25 \%-75 \%} \text { ) }\end{array}$ & 439.286 & $250.000-750.000$ \\
\hline Pobre Extremo & 30 & 30 \\
\hline Pobre No Extremo & 18 & 18 \\
\hline No Pobre & 52 & 52 \\
\hline \multicolumn{3}{|l|}{ Hacinamiento** } \\
\hline $\mathrm{Si}$ & 17 & 17 \\
\hline No & 83 & 83 \\
\hline \multicolumn{3}{|l|}{ Consulta en el Hospital } \\
\hline Primera vez & 3 & 3 \\
\hline Semanalmente & 9 & 9 \\
\hline Mensualmente & 52 & 52 \\
\hline Anualmente & 36 & 36 \\
\hline
\end{tabular}

*Encuesta Permanente de Hogares, 2013 (CBA: Costo de una Canasta Básica de Alimentos: zona urbana: $279.524 \mathrm{Gs} / \mathrm{mes}$ y para zona rural: $258.654 \mathrm{Gs} / \mathrm{mes}$; CBT Costo de una Canasta Básica Total: para zona urbana: $435.067 \mathrm{Gs} / \mathrm{mes}$ y para zona rural: $375.801 \mathrm{Gs} / \mathrm{mes}$ ). Para clasificación del nivel de pobreza: Ingreso Per cápita < CBA = pobre extremo; Ingreso Per cápita > CBA, pero, < CBT = pobre no extremo; Ingreso > CBA, $\mathrm{CBT}=$ no pobres (16).

**Dirección General de Estadística, Encuestas y Censos. Atlas de Necesidades Básicas Insatisfechas. 2005. Indicador de pobreza, y es considerado a partir de más de 3 individuos compartiendo el mismo dormitorio (17).

En cuanto a las características sexuales, el 54\% (54/100) inició sus relaciones sexuales a una edad igual o inferior a 17 años, el $80 \%(80 / 100)$ mantuvo relaciones sexuales con una persona dentro del último año. Además, una gran cantidad de mujeres $(58 \%, 58 / 100)$ no utilizaba preservativos en sus relaciones sexuales, el $90 \%$ de estas últimas (52/58) utilizaba métodos anticonceptivos diferentes al preservativo (anticonceptivos orales, inyectables, DIU y ligadura) y el $10 \%(6 / 58)$ restante no utilizaba ningún método anticonceptivo. También se observó que una importante proporción de ellas se realizó alguna prueba laboratorial para VIH $(67 \%, 67 / 100) 0$ sífilis $(63 \%, 63 / 100)$ previo al estudio. Ver Tabla 2. 
Tabla 2. Características sexuales de mujeres en edad fértil no gestantes. Hospital Regional de Villa Hayes. $n=100$

\begin{tabular}{|c|c|c|}
\hline Características & $\mathbf{n}$ & $\%$ \\
\hline Edad de la $1^{a}$ relación sexual (años) (mediana, $\mathrm{IC}_{25 \%-75 \%)}$ & 17 & $15-18$ \\
\hline$\leq 17$ & 54 & 54 \\
\hline$>17$ & 46 & 46 \\
\hline $\mathbf{N}^{\circ}$ de parejas sexuales en el último año (mediana, $\mathrm{IC}_{25 \%-75 \%}$ ) & 1 & $1-1$ \\
\hline 1 & 80 & 80 \\
\hline$\geq 1$ & 20 & 20 \\
\hline \multicolumn{3}{|l|}{ Uso de preservativos } \\
\hline Utiliza & 42 & 42 \\
\hline No utiliza* & 58 & 58 \\
\hline \multicolumn{3}{|l|}{ Análisis previo de VIH } \\
\hline $\mathrm{Si}$ & 67 & 67 \\
\hline No & 33 & 33 \\
\hline \multicolumn{3}{|l|}{ Análisis previo de sífilis } \\
\hline $\mathrm{Si}$ & 63 & 63 \\
\hline No & 37 & 37 \\
\hline
\end{tabular}

*Utiliza otros métodos anticonceptivos diferentes al preservativo (anticonceptivos orales, inyectables, DIU, ligadura) o no utiliza ningún método anticonceptivo.

Con respecto a los conocimientos, en general se encontró una alta frecuencia de respuestas correctas en todas las preguntas sobre prevención y transmisión de estas ITS. Las preguntas con mayor frecuencia de respuestas correctas fueron las que hacían referencia a los métodos anticonceptivos. Los resultados son mostrados en la Tabla 3 . El nivel de conocimiento por lo tanto fue alto y tan solo el $11 \%$ de las mujeres presentaron un nivel inaceptable. Ver Tabla 4.

En cuanto a los resultados a las pruebas serológicas utilizadas para la detección de anticuerpos dirigidos contra el Treponema pallidum, 14\% dieron resultado positivo con la prueba inmunocromatográfica, de los cuales 13 casos fueron confirmados con la prueba de inmunofluorescencia indirecta (FTA-ABS). Por el contrario, ningún resultado positivo para VIH se obtuvo por el método inmunocromatográfico y el método de ELISA.

Tabla 3. Conocimientos sobre prevención y transmisión de sífilis e infección por el VIH de las mujeres en edad fértil no gestantes. Hospital Regional de Villa Hayes. $n=100$

\begin{tabular}{|c|c|c|c|}
\hline & Si & No & Desconoce \\
\hline $\begin{array}{l}\text { Le han brindado información sobre la transmisión, prevención y tra- } \\
\text { tamiento de sífilis e infección por el VIH }\end{array}$ & 75 & 25 & --- \\
\hline Conoce los métodos anticonceptivos & 98 & 2 & --- \\
\hline $\begin{array}{l}\text { Puede reducirse el riesgo de transmisión de estas infecciones al man- } \\
\text { tener relaciones sexuales con una única persona fiel y no infectada }\end{array}$ & 78 & 13 & 9 \\
\hline $\begin{array}{l}\text { Puede reducirse el riesgo de transmisión de estas infecciones al utili- } \\
\text { zar preservativos }\end{array}$ & 80 & 15 & 5 \\
\hline Puede una persona de aspecto saludable tener VIH y sífilis & 71 & 15 & 14 \\
\hline Se transmiten estas infecciones por picadura de mosquitos & 15 & 69 & 16 \\
\hline $\begin{array}{l}\text { Se transmiten estas infecciones al compartir terere o mate con una } \\
\text { persona infectada }\end{array}$ & 26 & 68 & 6 \\
\hline
\end{tabular}


Tabla 4. Nivel de conocimiento de las mujeres en edad fértil no gestantes sobre prevención y transmisión de sífilis e infección por el VIH. Hospital Regional de Villa Hayes. $n=100$

\begin{tabular}{lll}
\hline Nivel de conocimiento & n & \% \\
\hline Inaceptable & 11 & 11 \\
Aceptable & 18 & 18 \\
Bueno & 19 & 19 \\
Muy bueno & 24 & 24 \\
Excelente & 28 & 28 \\
\hline
\end{tabular}

Se encontró asociación entre la menor cantidad de años de escolarización $(\leq 10)$ y la presencia de sífilis $(p=0,002)$. No se encontraron asociaciones entre la presencia de sífilis y edad de la primera relación sexual, número de parejas sexuales en el último año, uso de preservativos o conocimiento sobre estas ITS. Estos datos se muestran en la Tabla 5.

Tabla 5. Asociación entre las características socio-demográficas, socio-económicas y sexuales y presencia de sífilis. Hospital Regional de Villa Hayes. $n=100$

\begin{tabular}{|c|c|c|c|c|}
\hline \multirow{2}{*}{ Características } & \multirow{2}{*}{ Total } & \multicolumn{2}{|c|}{ Seropositivo para sífilis } & \multirow{2}{*}{ Valor $\mathbf{p}$} \\
\hline & & $\mathbf{n}$ & $\%$ & \\
\hline Edad (años) & & & & 0,052 \\
\hline$\leq 28$ & 52 & 3 & 6 & \\
\hline$>28$ & 48 & 10 & 21 & \\
\hline Estado civil & & & & 0,733 \\
\hline Casada o en pareja & 69 & 10 & 14 & \\
\hline Soltera o divorciada & 31 & 3 & 10 & \\
\hline Años de escolarización alcanzado & & & & 0,002 \\
\hline$\leq 10$ & 51 & 12 & 24 & \\
\hline$>10$ & 49 & 1 & 2 & \\
\hline Ubicación de la vivienda & & & & 0,864 \\
\hline Zona urbana & 75 & 10 & 13 & \\
\hline Zona rural & 25 & 3 & 12 & \\
\hline Hacinamiento & & & & 0,070 \\
\hline Vive en hacinamiento & 17 & 5 & 29 & \\
\hline No vive en hacinamiento & 83 & 8 & 10 & \\
\hline $\begin{array}{l}\text { Ingreso económico percápita } \\
\text { (guaraníes/mes) }\end{array}$ & & & & 1,000 \\
\hline$<439.286$ & 50 & 6 & 12 & \\
\hline$>439.286$ & 50 & 7 & 14 & \\
\hline Edad de la $1^{\circ}$ relación sexual (años) & & & & 0,774 \\
\hline$\leq 17$ & 54 & 8 & 15 & \\
\hline$>17$ & 46 & 5 & 11 & \\
\hline$N^{\circ}$ de parejas sexuales en el último año & & & & 0,503 \\
\hline 1 & 80 & 9 & 11 & \\
\hline$>1$ & 20 & 4 & 20 & \\
\hline Uso de preservativos & & & & 0,981 \\
\hline Utiliza & 42 & 5 & 12 & \\
\hline No utiliza & 58 & 8 & 14 & \\
\hline $\begin{array}{l}\text { Nivel de conocimiento sobre sífilis y } \\
\text { VIH }\end{array}$ & & & & 0,633 \\
\hline Aprobado & 89 & 11 & 12 & \\
\hline Reprobado & 11 & 2 & 18 & \\
\hline
\end{tabular}




\section{DISCUSIÓN}

En el Paraguay existen varios estudios que indican una alta frecuencia de ITS, entre ellas sífilis, infección por el VIH, en mujeres embarazadas (9-11), sin embargo, los datos en mujeres no gestantes son limitados (3). Por esta razón, el presente estudio tuvo como objetivo determinar la frecuencia de infección por el VIH, sífilis y los factores de riesgo asociados con la adquisición de estas infecciones.

La frecuencia de sífilis en mujeres no gestantes observada en este estudio (13\%) se encuentra por encima de la señalada por el PRONASIDA en mujeres gestantes, el cual estima que el $4,41 \%$ de las mujeres embarazadas en nuestro país presentan dicha infección (9). Así mismo es superior a la observada en mujeres gestantes residentes de Chile $(0,5 \%)$ y en las principales ciudades de Bolivia (3\%), registrado en el año 2.006 (5). La relativa alta frecuencia detectada pone de manifiesto la necesidad de realizar intervenciones en esta población a fin de evitar que las mujeres sufran complicaciones de la enfermedad como neurosífilis o transmitan al feto en los primeros meses de embarazo.

Esta alta frecuencia de sífilis observada se contrapone con el alto nivel de conocimiento sobre prevención y transmisión de sífilis e infección por el VIH de la población, principalmente en lo referente al conocimiento de los preservativos como método de protección contra las ITS (80\%), lo que concuerda con lo indicado en la Encuesta Nacional de Demografía y Salud Sexual y Reproductiva del año 2008, en donde se evidenció un alto conocimiento de preservativos en mujeres de 15 a 44 años de todo el país (12). Este nivel alto de conocimiento pudo ser debido a que en dicho hospital se realizan periódicamente charlas explicativas sobre las enfermedades más frecuentes en el país así como también sobre educación sexual y reproductiva, además el lugar cuenta con un departamento encargado de las ITS en donde el personal de salud se encarga de dar consejerías sobre estas infecciones a mujeres embarazadas y dar seguimiento a casos conocidos, en dichas consejerías se instruye y sugiere al paciente sobre el mejor método de prevención contra las ITS; y con un departamento de planificación familiar en donde se educa a las mujeres sobre los riesgos y beneficios de utilizar los métodos anticonceptivos y dejar a criterio la elección del método más adecuado.

En relación a los factores de riesgo analizados, se observó asociación entre la menor escolarización $(\leq 10)$ y la presencia de sífilis, lo que conlleva a pensar que la diseminación de esta infección está muy influenciada por el bajo nivel educativo según como un estudio de casos y controles realizado en México lo indica llevado a cabo en mujeres embarazadas (13), por lo que es de verdadera importancia fomentar el acceso a la educación en esta región. No se encontró asociación entre el uso de preservativos y la presencia de sífilis, aunque no se descarta la posibilidad de que hayan tenido otro tipo de ITS como gonorrea, virus del papiloma humano (VPH), entre otras que no estaban incluidos como objetivo de este estudio.

Cabe destacar que un alto porcentaje de la población incluyendo positivas y negativas para sífilis no utilizaba preservativos, lo cual en parte podría explicar la alta frecuencia de ITS observada.

El alto porcentaje de mujeres positivas para sífilis junto con el alto conocimiento que ellas tenían sobre prevención y transmisión de sífilis y la baja frecuencia de uso de preservativos sugieren que ellas obtuvieron información en algún momento sobre estas infecciones pero no comprendieron la importancia de prevención y no dimensionaron el riesgo al que ellas están expuestas.

Es interesante resaltar que la baja frecuencia de uso de preservativos (42\%) pudo deberse a que la mayoría de estas mujeres optaban por otros métodos anticonceptivos diferentes con el objetivo de reducir la probabilidad de embarazo antes que un método de protección contra las ITS y/o por el rechazo de sus parejas al uso de preservativos 
manifestado por las mujeres encuestadas. Esto considerando que en el Hospital de Villa Hayes distribuían gratuitamente a las mujeres y a sus parejas preservativos masculinos.

No se encontró asociación entre el número de parejas sexuales en el último año y la presencia de sífilis. A pesar que la mayoría de las mujeres declararon tener solo una pareja sexual, no puede descartarse que en el presente estudio no fue tomado datos sobre el número de parejas sexuales que tenían los esposos o concubinos, que podría ser uno de los factores de riesgo que en parte explique la alta frecuencia de sífilis detectada.

Tampoco, se observó asociación entre la edad de la primera relación sexual y la presencia de sífilis. Estudios realizados sobre el virus del papiloma humano (VPH), que al igual que sífilis al ser de transmisión sexual comparten co-factores de riesgo de adquisición de infección, sugieren que la edad de inicio de relaciones sexuales es una predictora del número de parejas sexuales, ya que al tener un inicio temprano de las relaciones sexuales, mayor es la probabilidad de tener un mayor número de parejas sexuales $(14,15)$. Sin embargo, en este caso la mayoría de las mujeres declararon poseer solo una pareja sexual. Al parecer otros co-factores de riesgo de adquisición de ITS podrían estar influenciando, como el número de parejas sexuales que presenta su pareja.

Además no se encontró asociación entre el nivel de conocimiento sobre prevención y transmisión de la infección por el VIH y sífilis, y la presencia de esta última infección, probablemente esto pudo ser debido a que las mujeres encuestadas tenían buenos conocimientos acerca de estas infecciones, no así buenos comportamientos sexuales, por ejemplo la falta de uso de preservativos.

A nivel nacional, el PRONASIDA registró una frecuencia de infección por el VIH de $0,5 \%$ en mujeres embarazadas en el 2.013 (3). En este estudio no se observaron casos de infecciones por el VIH, esto podría deberse al número limitado de mujeres tomadas en el estudio. Por tanto para tener datos más certeros sobre la frecuencia de infección por el VIH es preciso aumentar el tamaño de muestra.

A pesar de ser un estudio que incluye población hospitalaria que podría presentar sesgos, se observó una concordancia entre los resultados obtenidos en el presente trabajo y las características sexuales observadas en registros del Centro Paraguayo de Estudios de Población (CEPEP) a través de la Encuesta Nacional de Demografía y Salud Sexual y Reproductiva (12) y en el trabajo de Mendoza et al., 2013 (6). El CEPEP publicó en el año 2.008 una edad de inicio de relaciones sexuales de 18,2 años en mujeres de 15 a 44 años de edad en 4 regiones del país que incluían Asunción y Área Metropolitana, Norte, Centro Sur y el Este; además en dicha publicación se observó un aumento en el número de mujeres que tuvieron su primera relación sexual antes de los 20 años de edad en comparación a años anteriores (12), dato que debería de llamar la atención como para incentivar más campañas de educación sexual a nivel nacional. En cuanto al número de parejas sexuales en el último año, se encontró una mediana de 1 pareja sexual por mujer, concordando con un estudio realizado en el mismo departamento en mujeres indígenas no gestantes (6).

En conclusión, la alta frecuencia de sífilis observada y el bajo porcentaje de mujeres que utilizaban preservativo como método anticonceptivo de preferencia, que se contrapone al elevado nivel de conocimiento sobre prevención y transmisión de sífilis e infección por el VIH sugieren que es preciso fortalecer la detección de ITS en este grupo etáreo, así como las campañas de educación que enfaticen la importancia de realizar buenas prácticas sexuales a fin de mejorar las condiciones de vida de esta población.

Agradecimientos. A las Dras. Raquel Aguilar, Celia Olmedo, Karen Quiñónez, Elena Insfrán, Carolina Ruíz y el Dr. Victorino Noguera, a la Licenciada Martha Marecos, y a las Técnicas de Laboratorio Amanda Núñez y Nahir Núñez quienes acompañaron la fase de 
reclutamiento y parte de la fase analítica llevadas a cabo en el Hospital Regional de Villa Hayes. A la Dra Maria Mercedes Carpinelli y al Dr. Enrique Granado, quienes apoyaron en parte de la fase analítica llevada a cabo en el Instituto de Investigaciones en Ciencias de la Salud (IICS). A la empresa GT Scientific y al Director de esta empresa, el Dr. Felipe Sanabria, por el aporte de parte de los reactivos necesarios para la fase analítica.

\section{REFERENCIAS BIBLIOGRAFICAS}

1. Ortayli N, Ringheim K, Collins L, Sladden T. Sexually transmitted infections: Progress and challenges since the 1994. International Conference on Population and Development (ICPD) Contraception. 2014;90 (6):S22-S31.

2. Johnson LF, Dorrington RE, Bradshaw D, Coetzee DJ. The role of sexually transmitted infections in the evolution of the South African HIV epidemic. Trop Med Int Health. 2012;17(2):161-8.

3. Ministerio de Salud Pública y Bienestar Social (MSPyBS), Programa Nacional de Control del VIH/SIDA/ITS. Informe de la situación epidemiológica del VIH/sida y sífilis Paraguay /Internet/. MSPyBS; 2013. [citado 12 Ago 2014]. Disponible en: http://www.mcp.org.py/documentos/ situacion_epidemiologica1314.pdf

4. Aguayo N. Estudio de prevalencia del VIH/ Sífilis y comportamientos, prácticas y actitudes de las mujeres trabajadoras sexuales en el Paraguay 2011-2012 [Internet]. Asunción: Unidas en la Esperanza (UNES); 2012. [citado 7 May 2015]. Disponible en: http://www.pronasida.gov.py/images/ documentos/mts_2011_2012.pdf.

5. Galban E, Benzaken AS. Situación de la sífilis en 20 países de Latino América y el Caribe: Año 2006. DST-J bras Doenças Sex Transm. 2007;19(3-4):166-72.

6. Mendoza L, Mongelos P, Páez M, Castro A, Rodriguez Riveros I, Gimenez G, et al. Human papillomavirus and other genital infections in indigenous women from Paraguay: a cross-sectional analytical study. BMC Infect Dis. 2013;13:531.

7. WHO. Reproductive health indicators. Guidelines for their generation, interpretation and analysis for global monitoring. Geneva: WHO; 2006.

8. Rodríguez-Riveros $\mathrm{MI}$, Insaurralde Alviso $\mathrm{A}$, Páez M, Mendoza L, Castro A, Giménez G. Conocimientos, actitudes y prácticas sobre sífilis materna: Adolescentes embarazadas. Hospitales de referencia en Paraguay. 2008. Mem. Inst. Investig. Cienc. Salud. 2012;10(1):76-84.
9. Ministerio de Salud Pública y Bienestar Social. Programa Nacional de Control del VIH/ITS. Estudio de prevalencia de sífilis y VIH en parturientas y puérperas en el Paraguay 2012 [Internet]. PRONASIDA; 2012. [citado $1 \mathrm{mar}$ 2015]. Disponible en: http:// www.pronasida.gov.py/images/documentos/ material_educativo/inf_est_puerperas.pdf.

10.Páez M, Riveros MI. Situación epidemiológica de la sífilis materna y congénita en el sub sector público a nivel nacional, Paraguay - 20002004. DST-J bras Doenças Sex Transm. 2006;18(2):117-23.

11.Leguizamón R, Vega ME. Epidemiología de VIH/SIDA en el Hospital Nacional de Itauguá, Paraguay. Rev. Nac. (Itauguá). 2012;4(1):2733.

12. Centro Paraguayo de Estudios de Población. Encuesta nacional de demografía y salud sexual y reproductiva 2008: Informe final [Internet]. Asunción: CEPEP; 2009. [citado 4 mar 2015]. Disponible en: http:// pdf.usaid.gov/pdf_docs/PNADR811.pdf.

13.González-Pacheco I, Lartigue T, Vázquez G. Estudio de casos y controles en un grupo de mujeres embarazadas con experiencias adversas en la infancia y/o adolescencia e infecciones de transmisión sexual. Salud Ment. 2008;31(4):261-70.

14.Burchell AN, Winer RL, de Sanjosé S, Franco EL. Chapter 6: Epidemiology and transmission dynamics of genital HPV infection. Vaccine. 2006;24(suppl 3):S52-S61.

15. Hildesheim A, Herrero R, Castle PE, Wacholder $S$, Bratti MC, Sherman ME, et al. HPV cofactors related to the development of cervical cancer: results from a population-based study in Costa Rica.. Br J Cancer. 2001;84(9):121926.

16.Dirección General de Estadística, Encuestas y Censos (DGEEC). Principales resultados de pobreza y distribución del ingreso 2013 / Internet/. Fernando de la Mora: DGEEC; 2013. [citado 2 mar 2015]. Disponible en: http:// www.dgeec.gov.py/Publicaciones/Biblioteca/ eph 2014 /Boletin $\% 20$ de $\% 20$ pobreza\% 202014.pdf 
17.Dirección General de Estadística, Encuestas y Censos. Atlas de necesidades básicas insatisfechas /Internet/. Fernando de la Mora: DGEEC; 2005. [citado 2 mar 2015]. Disponible en: http://www.dgeec.gov.py/ Publicaciones/Biblioteca/Atlas\%20NBI/0a\% 20NBI\%20Presentacion.pdf 